\title{
Progressive multifocal leukoencephalopathy, a possible complication in a patient with bladder cancer
}

\author{
S. Latifyan ${ }^{1,2 *}$, S. Sideris ${ }^{1,2}$, A. Papaleo ${ }^{1}$, L. Divano ${ }^{1,3}$, H. Visee ${ }^{1,3}$, M. Malarme ${ }^{1,3}$, A. Georgala ${ }^{1,2}$ and D. De Bels ${ }^{1,3}$ \\ ${ }^{1}$ Institut Jules Bordet, Brussels, Belgium \\ ${ }^{2}$ Université Libre de Bruxelles, Brussels, Belgium \\ ${ }^{3}$ Centre Hospitalier Universitaire Brugmann, Brussels, Belgium
}

\begin{abstract}
Progressive multifocal leukoencephalopathy (PML) is a demyelinating disease of the brain, caused by reactivation of the polyomavirus JC (JCV). PML has classically been described in individuals with profound cellular immunosuppression such as patients with AIDS, hematological malignancies, organ transplant recipients or those treated with immunosuppressive or immunomodulatory medications. The occurrence of PML in patients without identifiable immunodeficiency represents a therapeutic challenge. Tumor-induced immune suppression is a fundamental problem in cancer biology. We present a case of 71-year-old man with bladder cancer. The patient was diagnosed with PML after an epileptic seizure followed by a coma. MRI images were compatible with PML. Presence of JC virus in the cerebrospinal fluid confirmed the diagnosis. Unfortunately the patient died shortly after the diagnosis. There is no specific treatment for JCV. The reconstitution of the immune system seems the most effective approach.
\end{abstract}

\begin{abstract}
Abbreviations: PML: Progressive Multifocal Leukoencephalopathy; JCV: John Cunningham Virus; AIDS: Acquired Immune Deficiency Syndrome; CSF: Cerebrospinal Fluid; HIV: Human Immuno deficiency Virus; HSV: Herpes Simplex Virus; VZV: Varicella Zoster Virus; CMV: Cytomegalovirus; ADC: Apparent Diffusion Coefficient; SSPE: Subacute Sclerosing Pan Encephalopathy; MRI: Magnetic Resonance Imaging; HAART: Highly Active Antiretroviral Therapy
\end{abstract}

\section{Introduction}

Progressive multifocal leukoencephalopathy (PML) is a rare, progressive and lethaldemyelinating disease of the brain, causing irreversible neurological damages [1]. It is caused by the John Cunningham virus (JCV), a Polyomavirus, responsible for the destruction of oligodendrocytes. Most humans have been exposed to the JC virus during their lifetimes, and infection typically occurs during the first several decades of life; approximately $50-80 \%$ of adults have serologic evidence of prior exposure. Following initial infection, the virus remains latent in multiple tissues in healthy individuals.

Reactivation of JC virus due to reduction of cellular immunity is the most common underlying cause. PML has classically been described in individuals with profound cellular immunosuppression such as patients with acquired immune deficiency syndrome (AIDS) - $82 \%$, hematological malignancies - $8 \%$, organ transplant recipients - $3 \%$, rheumatology pathologies $-0.44 \%$ or patients with autoimmune disease under immunosuppressive or immunomodulatory treatment [2].

The PML is very rare in case of solid tumors. The clinical presentation varies depending on the location and the extent of the affected cerebral area. For the moment there is no specific treatment.

\section{Case report}

We present the case of a 71 year-old male patient hospitalized for asthenia, abdominal pain, back pain and macroscopic hematuria. The physical examination revealed positive lumbar point bilaterally whereas the neurological examination was normal. The biology was in favor of an inflammatory syndrome, anemia and acute renal failure. The abdominal tomography showed a bladder tumor with central necrosis and right uretero-hydronephrosis. The further investigations revealed a deep venous thrombosis of the right lower limb and massive pulmonary embolism requiring therapeutic anticoagulation.

A month after the diagnosis of bladder cancer, the patient was treated by partial endoscopic tumour resection. The anatomopathological result revealed a stage pT3 high grade urothelial carcinoma. The patient was elected for systemic neo-adjuvant chemotherapy. The initial treatment was a single dose Mitomycin C instillation. The general status of the patient excluded further systemic treatment with cisplatin based chemotherapy regimen. Shortly after the local treatment the patient suffered a grand mal epileptic seizure. The electroencephalogram revealed unmoral brain activity especially in the left temporal and occipital lobe (Figure 1). The cerebral magnetic resonance was suggestive for diffuse encephalopathy showing decreased signal of the periventricular white substance on $\mathrm{T} 1$ with contrast appearance suggestive of diffuse encephalopathy particularly centered at the white substance (Figure 2). Further investigations were not possible at this time as the patient signed a discharge and left the hospital.

Three days later the patient presented with acute respiratory distress

Correspondence to: S. Latifyan, Université Libre de Bruxelles, Brussels, Belgium E-mail: sofia.latifyan@bordet.be

Keywords: progressive multifocal leukoencephalopathy, bladder cancer, tumor induced immunosuppression

Received: October 02, 2015; Accepted: October 20, 2015; Published: October 23,2015 


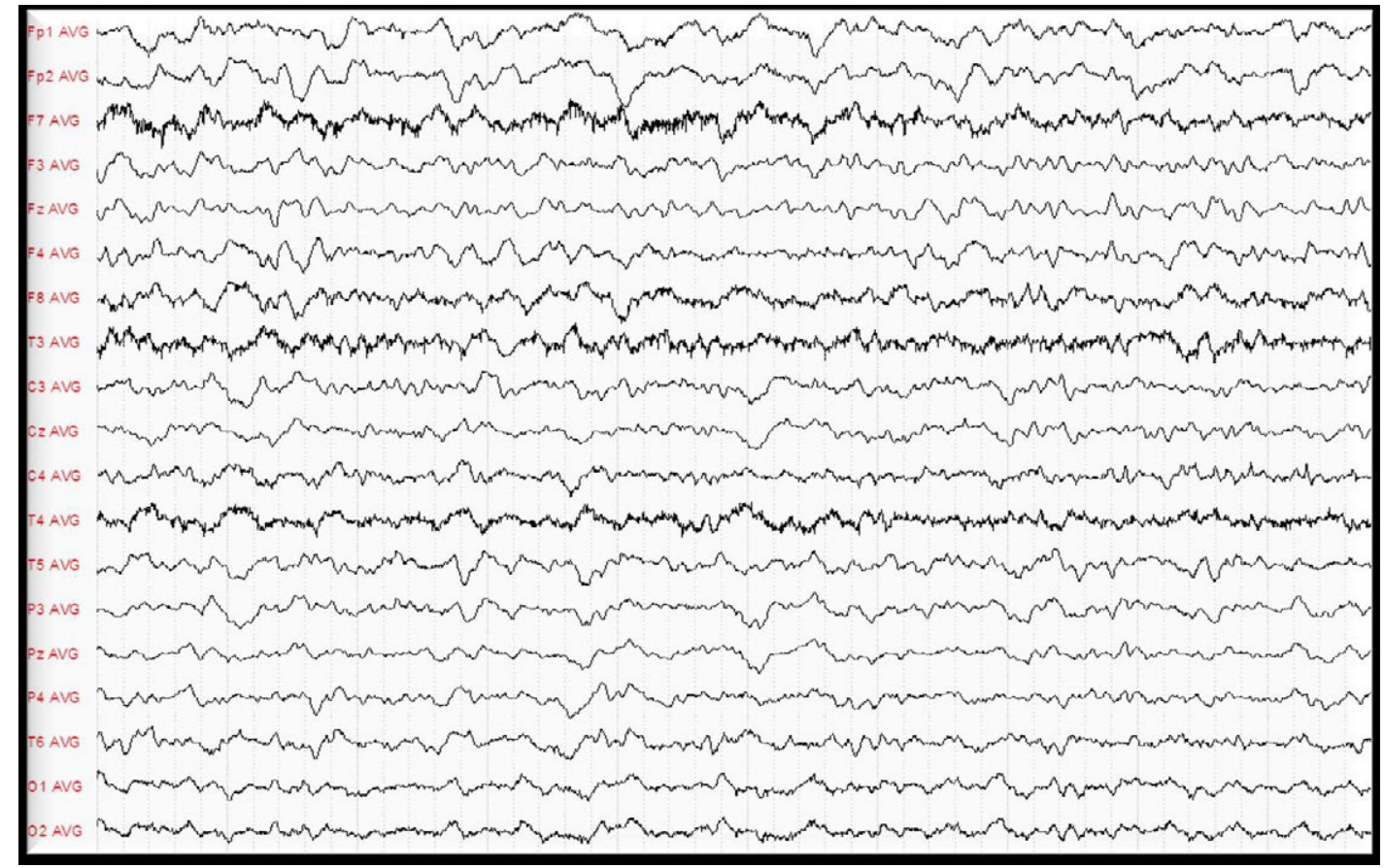

Figure 1. EEG.

The exam revealed abnormal brain activity especially in the left temporal and occipital lobe.
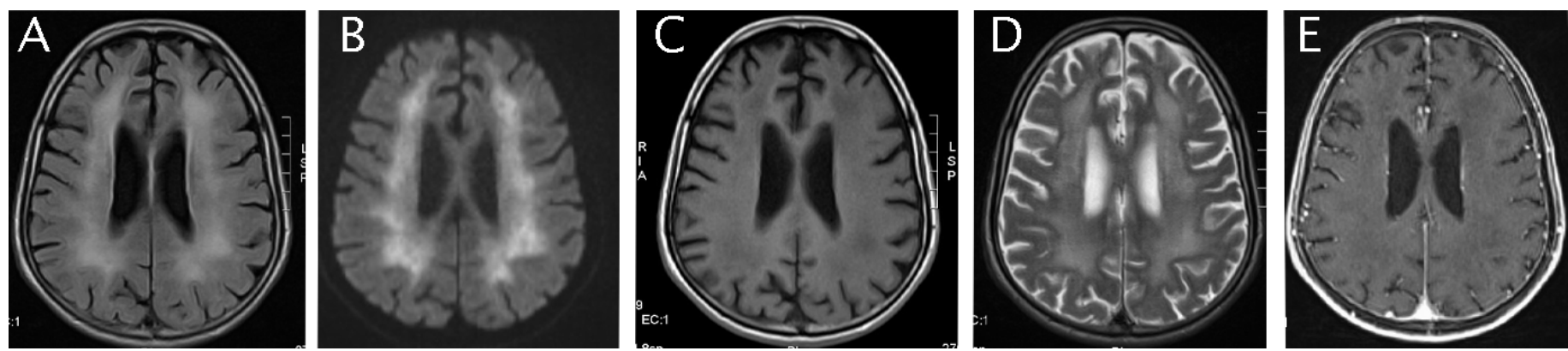

Figure 2. All are MRI axial images.

A: Enhancement of the periventricular white mater on a flair sequence. B: White mater hypersignal on a diffusion sequence associated with a restricted diffusion coefficient. C: Diffuse white mater decreased signal on T1-weighted images. D: Diffuse white mater hypersignal on T2-weighted images. E: Absence of contrast-enhanced images.

and pyrexia due to underlying pneumonia and simultaneous urinary infection. He was hospitalized in the intensive care unit and received adequate antibiotic therapy and hydration. However his general status was marked by a progressive loss of consciousness leading to a coma. Results of cerebrospinal fluid (CSF) cellular, biochemical, and routine microbiology studies were within normal limits, but the real time quantitative PCR, was positive for JCV, confirming the diagnosis of viral encephalopathy. Further laboratory tests didn't show any markers of immunospression. The complementary serology analysis excluded infections of human immunodeficiency virus (HIV), Borrelia, herpes simplex virus (HSV), varicella zoster virus (VZV), cytomegalovirus (CMV) or Enterovirous. The patient died shortly after the diagnosis. An autopsy was refused by the family.

\section{Discussion}

Progressive multifocal leukoencephalopathy (PML) was first described in 1958 in two patients with hematologic malignancies. In early 80 s the incidence rate of PML increased significantly following the HIV epidemic expansion. Today PML together with toxoplasmosis and pneumocystis carinii, is considered as one of the most common opportunistic infections for patients with impaired immune system [3]. PML is caused by the JC virus which belongs to the type Polyomavirus, of the family of Papovariridae [4].The viral particles, causing the illness, in oligodendrocytes have been identified in 1965. Six years later, in 1971, the virus was isolated from a cerebral sample coming from a man attained by PML and named by the initials of the patient - John Cunningham.

The JC virus is in the environment. About $80 \%$ of the population has been displayed to the virus before attaining the medium age. It resides during years in a sleeping state in the kidneys, the bone marrow and the lymphatic ganglions. Ordinarily, the virus is reactivated only in $2 \%$ to $6 \%$ of the persons attained by the AIDS, notably those having less than 100 cells CD4 [5]. The prevalence of PML is $0.07 \%$ for patients with hematologic malignancies [6] $0.8 \%$ for liver transplant recipients [7] and 1.2/1000 for patients with heart or/and lung transplantation [8]. 
Gheuens et al. performed a review of the literature to find cases of PML in individuals with minimal or occult immunosuppression. Of a total of 38 cases, 7 (18.4\%) had hepatic cirrhosis, $5(13.2 \%)$ had renal failure, $2(5.2 \%)$ were pregnant women, $2(5.2 \%)$ had concomitant dementia, 1 (2.6\%) had dermatomyositis and $22(57.9 \%)$ had no specific underlying diagnosis. The outcome was fatal in $27 / 38$ (71.1\%) cases within 1.5120 months (median 8 months) from onset of symptoms, and $3 / 4$ cases who harbored JCV-specific T cells in their peripheral blood had inactive disease with stable neurological deficits after 6-26 months of follow-up [9].

Primary JC infection is usually asymptomatic whereas in patients developing the disease the clinical presentation is associated to the location and the extent of the affected cerebral area. JC virus induces apoptosis of oligodendrocytes however the molecular basis of this phenomena remains unclear. Traditionally there are motor deficit, cognitive disturbances, dysarthria and dysphasia, headache, disturbances of the step, affection of the visual field and loose of the sensibility. The clinical state of the patient deteriorates in a progressive and uninterrupted way up to the death.

Cerebral magnetic resonance (MRI) is the golden standard for the detection of brain lesions, however not JC specific. They usually involve periventricular and subcortical white mater, are bilateral and do not take contrast. Lesions have a decreased signal on T1-weigthed images and an increased signal on T2-weighted images. There is normally no mass effect [10]. In the case of our patient the cerebral MRI imposed two diagnostic hypotheses. The first was for a toxic origin. In this case there is a spongy degeneration of the white cerebral substance and intramyelique edema which explains the diffuse hyper signal and decreased apparent diffusion coefficient (ADC). The second hypothesis was of a subacute sclerosing pan encephalopathy (SSPE). This pathology is rare and usually follows a viral infection possibly after a clinically latent period of months or years.

The detection of the viral DNA in the CSF is the best available way of non-invasive diagnosis. The technique is characterized by a sensitivity of $75 \%$ and a specificity of $96 \%$ [11]. A low JC concentration in the CSF (50-100 copies per $\mathrm{ml}$ ) is associated with better outcome [12]. The detection of JCV within oligodendrocytes can be accomplished by in situ hybridization or immunohistochemistry but requires performing of a brain biopsy which is almost abandoned (high morbid-mortalty) with the advent of molecular biology techniques combined with MRI [13]. It is currently accepted that the diagnosis of PML can be made in any clinical and radiological suggestive context with positive JCV DNA in the CSF in immunocompromised patients.

Therewerenoobjectivebiologicalarguments of immunosuppression in the case of our 71 years old patient with local treatment for pT3 urothelial cancer. Nevertheless, tumor-induced immune suppression is a fundamental problem in cancer biology; Cancer cells display multiple immunosuppressive mechanisms to evade T-cell responses, either to avoid immune recognition or to disable effector T-cells. It has been proposed that the tumor microenvironment can restrict the effectiveness of anti-tumor responses by displaying a variety of immunosuppressive strategies [14-16]. These include alterations of components of the antigen presentation machinery, defects in proximal TCR signaling, secretion of immunosuppressive and proapoptotic factors, activation of negative regulatory pathways and specific recruitment of regulatory cell populations $[14,17-20]$.

There is no specific treatment for JCV. In the context of immunodefiency syndrome, numerous drugs have been tried empirically such as interferon alpha-2, cytarabine, cidofovir and topotecan [21] with no evident benefit. An open label study involving 19 immune competent PML patients showed that intravenous cytarabine $2 \mathrm{mg} / \mathrm{kg} /$ day for five consecutive days seemed to stabilize $36 \%$ of patients up to four and a half despite a significant bone marrow toxicity [22]. Cytarabine was disappointing in vivo probably because of its poor bone penetration. Camptothecin derivatives, such as topotecan, topoisomerase I inhibitors were considered too toxic whereas cidofovir, a nucleotide analogue active against DNA viruses, was ineffective in one international meta-analysis [23]. Further treatments using HT2A serotoninergic receptor antagonists or immunomodulators are still under evaluation. In HIV patients with PML, optimization or initiation of highly active antiretroviral therapy (HAART) is the best therapeutic option. Patients treated with HAART therapy have a one-year survival rate of about 50 percent, compared with approximately 10 percent in those not treated with HAART therapy [24].

\section{Conclusion}

Progressive multifocal leukoencephalopathy occurs almost exclusively in immunosuppressed individuals. Only few cases of immune competent patients with PML have been reported. Tumorinduced immune suppression is a fundamental problem in cancer biology.

Our case suggests that a possibly mildly immunocompromised host does not exclude the diagnosis of PML. The diagnosis should be considered based on the clinic and the MRI findings and confirmed by the detection of the JC virus by a lumbar puncture.

Based on the fact that there is no specific treatment for PML, the reconstitution of the immune system seems the most effective approach especially in case of HIV patients. Further molecular studies should be conducted in order to understand the pathogenesis of PML and proceed to the development of specific antiviral drugs.

\section{Competing interest}

We all have no competing interests related with that article to declare.

\section{References}

1. Koralnik IJ (2006) Progressive multifocal leukoencephalopathy revisited: Has the disease outgrown its name? Ann Neurol 60: 162-173. [Crossref]

2. Gupta M, Batra A, Patidar Y, Chowdhury D, Khwaja GA, et al (2012) Progressive multifocal leukoencephalopathy in an immunocompetent patient: A case report Neurology Asia 17: 67-69.

3. El Khattabi L, Patey O, Breuil J, et al. (2008) JC virus et Leukoencephalopathy multifocal progressive. Practicaterapeutica Anul XII12.

4. Frisque RJ, Bream GL, Cannella MT (1984) Human polyomavirus JC virus genome. $J$ Virol 51: 458-469. [Crossref]

5. Leukoencephalopathy multifocal progressive (LMP) - La source canadienne de renseignementssur le VIH etHÃ Cpatite C.

6. Power C, Gladden JG, Halliday W, Del Bigio MR, Nath A, et al. (2000) AIDS- and non-AIDS-related PML association with distinct p53 polymorphism. Neurology 54: 743-746. [Crossref]

7. Martinez AJ, Ahdab-Barmada M (1993) The neuropathology of liver transplantation: comparison of main complications in children and adults. Mod Pathol 6: 25-32. [Crossref]

8. Mateen FJ, Muralidharan R, Carone M, van de Beek D, Harrison DM, et al. (2011) Progressive multifocal leukoencephalopathy in transplant recipients. Ann Neurol 70: 305-322. [Crossref] 
9. Gheuens S, Pierone G, Peeters P, Koralnik IJ (2010) Progressive multifocal leukoencephalopathy in individuals with minimal or occult immunosuppression. $J$ Neurol Neurosurg Psychiatry 81: 247-254. [Crossref]

10. Whiteman ML, Post MJ, Berger JR, Tate LG, Bell MD, et al. (1993) Progressive multifocal leukoencephalopathy in $47 \mathrm{HIV}$-seropositive patients: neuroimaging with clinical and pathologic correlation. Radiology 187: 233-240. [Crossref]

11. Cinque P, Scarpellini P, Vago L, Linde A, Lazzarin A (1997) Diagnosis of centra nervous system complications in HIV-infected patients: cerebrospinal fluid analysis by the polymerase chain reaction. AIDS 11:1-17. [Crossref]

12. Yiannoutsos CT, Major EO, Curfman B, Jensen PN, Gravell M, et al. (1999) Relation of JC virus DNA in the cerebrospinal fluid to survival in acquired immunodeficiency syndrome patients with biopsy-proven progressive multifocal leukoencephalopathy. Ann Neurol 45:816-21. [Crossref]

13. Skolasky RL, Dal Pan GJ, Olivi A, Lenz FA, Abrams RA, et al. (1999) HIV-associated primary CNS lymorbidity and utility of brain biopsy. J Neurol Sci 163: 32-38. [Crossref]

14. Drake CG, Jaffee E, Pardoll DM (2006) Mechanisms of immune evasion by tumors. Adv Immunol 90: 51-81. [Crossref]

15. Igney FH, Krammer PH (2002) Immune escape of tumors: apoptosis resistance and tumor counterattack. J Leukoc Biol 71: 907-920. [Crossref]

16. Whiteside TL1 (2006) Immune suppression in cancer: effects on immune cells, mechanisms and future therapeutic intervention. Semin Cancer Biol 16: 3-15. [Crossref]
17. Steinman RM, Hawiger D, Nussenzweig MC (2003) Tolerogenic dendritic cells. Annu Rev Immunol 21: 685-711. [Crossref]

18. Khong HT, Restifo NP (2002) Natural selection of tumor variants in the generation of "tumor escape" phenotypes. Nat Immunol 3: 999-1005. [Crossref]

19. Gerlini G, Tun-Kyi A, Dudli C, Burg G, Pimpinelli N, et al. (2004) Metastatic melanoma secreted IL-10 down-regulates CD1 molecules on dendritic cells in metastatic tumo lesions. Am J Pathol 165: 1853-1863. [Crossref]

20. Blank C, Gajewski TF, Mackensen A (2005) Interaction of PD-L1 on tumor cells with PD-1 on tumor-specific T cells as a mechanism of immune evasion: implications for tumor immunotherapy. Cancer Immunol Immunother 54: 307-314. [Crossref]

21. Koralnik IJ, Gonzalez-Scarano F, Dashe JF (2015) Progressive multifocal leukoencephalopathy: Prognosis and treatment.

22. Aksamit AJ (2001) Treatment of non-AIDS progressive multifocal leukoencephalopathy with cytosine arabinoside. J Neurovirol 7: 386-390. [Crossref]

23. Gasnault J, Taoufik Y (2006) New trends in progressive multifocal leukoencephalopathy. Rev Neurol (Paris) 162: 43-56. [Crossref]

24. Antinori A, Cingolani A, Lorenzini P, Giancola ML, Uccella I, et al. (2003) Clinica epidemiology and survival of progressive multifocal leukoencephalopathy in the era of highly active antiretroviral therapy: data from the Italian Registry Investigative Neuro AIDS (IRINA). J Neurovirol 9: 47. [Crossref]

Copyright: (C2015 Latifyan S. This is an open-access article distributed under the terms of the Creative Commons Attribution License, which permits unrestricted use, distribution, and reproduction in any medium, provided the original author and source are credited. 